\title{
Radiosensitizing effect of galunisertib, a TGF-ß receptor I inhibitor, on head and neck squamous cell carcinoma in vitro
}

\author{
Bernhard J. Jank ${ }^{1,2}$ - Teresa Lenz ${ }^{1} \cdot$ Markus Haas $^{1} \cdot$ Lorenz Kadletz-Wanke $^{1} \cdot$ Nicholas J. Campion $^{1} \cdot$ Julia Schnoell $^{1}$. \\ Gregor Heiduschka ${ }^{1} \cdot$ Karin Macfelda ${ }^{2}$
}

Received: 7 October 2021 / Accepted: 24 December 2021 / Published online: 5 January 2022

(c) The Author(s) 2022

\begin{abstract}
Summary
Background. Resistance to radiation therapy poses a major clinical problem for patients suffering from head and neck squamous cell carcinoma (HNSCC). Transforming growth factor $\beta$ (TGF- $\beta$ ) has emerged as a potential target. This study aimed to investigate the radiosensitizing effect of galunisertib, a small molecule TGF- $\beta$ receptor kinase I inhibitor, on HNSCC cells in vitro. Methods. Three HNSCC cell lines were treated with galunisertib alone, or in combination with radiation. Of those three cell lines, one has a known inactivating mutation of the TGF-ß pathway (Cal27), one has a TGF- $\beta$ pathway deficiency $(\mathrm{FaDu})$ and one has no known alteration (SCC-25). The effect on metabolic activity was evaluated by a resazurin-based reduction assay. Cell migration was evaluated by wound-healing assay, clonogenic survival by colony formation assay and cell cycle by FACS analysis. Results. Galunisertib reduced metabolic activity in FaDu, increased in SCC-25 and had no effect on CAL27. Migration was significantly reduced by galunisertib in all three cell lines and showed additive effects in combination with radiation in CAL27 and SCC-25. Colony-forming capabilities were reduced in SCC-25 by galunisertib and also showed an additive effect with adjuvant radiation treatment. Cell cycle analysis showed a reduction of cells in $\mathrm{G}_{1}$ phase in response to galunisertib treatment. Conclusion. Our results indicate a potential antineoplastic effect of galunisertib in HNSCC with intact TGF-ß signaling in combination with radiation.
\end{abstract}

Keywords Head and neck squamous cell carcinoma $\cdot$ Galunisertib $\cdot$ Radiosensitivity $\cdot$ TGF- $\beta$

\section{Abbreviations \\ HNSCC Head and neck squamous cell carcinoma \\ TGF- $\beta \quad$ Transforming growth factor $\beta$}

\section{Background}

Head and Neck Squamous Cell Carcinoma (HNSCC) is the seventh most common type of cancer worldwide, affecting more than 5.5 million people, causing over 380,000 deaths

Gregor Heiduschka and Karin Macfelda these authors contributed equally to this work.

Bernhard J. Jank

bernhard.jank@meduniwien.ac.at

1 Department of Otorhinolaryngology, Head and Neck Surgery, Medical University of Vienna, Waehringer Guertel 18-20, 1090 Vienna, Austria

2 Department of Biomedical Research, Medical University of Vienna, Waehringer Guertel 18-20, 1090 Vienna, Austria every year [1-3]. HNSCCs represents a group of tumors that can arise from the mucosa of the nasal or oral cavity, the pharynx or larynx. Despite multidisciplinary treatment approaches, advanced HNSCC has a 5-year overall survival rate of only $\sim 50 \%$ [4]. Radiotherapy is an important cornerstone in the treatment of HNSCC patients, either as adjuvant therapy after surgery or as definitive radiochemotherapy in patients unfit for surgery or with locally advanced disease. Resistance to radiation is therefore particularly associated with a poor prognosis and represents a major clinical problem [5]. Strikingly, only the epidermal growth factor receptor antibody cetuximab has been approved as a radiosensitizing agent in the last 50 years [6].

Radioresistance is defined as either no or only partial response of the tumor to radiation therapy or early recurrence within a few weeks after initial response [5]. The biological mechanisms are complex and involve, among others, the Transforming Growth Factor (TGF) pathway with TGF- $\beta$ as one of its key members [7]. Although TGF- 3 has tumor-suppressing functions in healthy cells and most earlystage cancers, its activation in late-stage disease can promote 
tumorigenesis [8]. Notably, increased TGF- $\beta$ expression has been found in $80 \%$ of HNSCC and TGF- $\beta$ expression levels correlate with more advanced disease and worse patient survival [9].

The experimental cancer drug galunisertib (LY2157299) is a small molecule inhibitor of the TGF- $\beta$ receptor I and has shown antineoplastic effects in various cancer entities in vitro and in vivo [10]. Furthermore, galunisertib is currently being tested in cancer clinical trials for several different cancers, highlighting the expected potential of this novel drug [11]. To date, however, no data exist on the effect of galunisertib on HNSCC. The aim of this study was, therefore, to preclinically evaluate the effect of galunisertib in single- and combination treatment with radiation on head and neck squamous cell carcinoma in vitro.

\section{Methods}

\section{Cells and reagents}

HNSCC cell lines (FaDu, SCC-25 and Cal 27) were purchased from American Type Culture Collection (ATCC, Manassas, Virginia, USA) and cultured in Dulbecco's Modified Eagle Medium supplemented with 10\% FBS (Life Technologies) and 1\% Penicillin-Streptomycin (Thermo Fisher, Waltham, Massachusetts, U.S.) on standard cell-culture plastic. Cells were cultured under standard culture conditions $\left(37{ }^{\circ} \mathrm{C}\right.$ in $\left.5 \% \mathrm{CO} 2\right)$ and passaged with $0.05 \%$ trypsin/ EDTA at $80 \%$ confluency. Galunisertib (LY2157299) was purchased from Selleck Chemicals (Houston, Texas, USA), dissolved in DMSO as stock solution following the manufacturer's instructions and stored at $-20{ }^{\circ} \mathrm{C}$ until use. The final dilution in culture medium was kept below $0.16 \%$ DMSO concentration. The same concentration of DMSO was added in untreated controls as a vector control.

\section{Irradiation}

Cell lines were irradiated at a dose of $1.0 \mathrm{~Gy} / \mathrm{min}$ at a fixedfocus object distance of $45.5 \mathrm{~cm}$. A $200 \mathrm{kV}$ YXLON Maxishot X-ray unit (Yxlon International X-ray GmbH, Hamburg, Germany) with a tube current of $20 \mathrm{~mA}$ and a focus size of $5.5 \mathrm{~mm}, 4 \mathrm{~mm} \mathrm{Al}$ and $0.6 \mathrm{~mm} \mathrm{Cu}$ filter served as the radiation source.

\section{Metabolic activity assay}

To evaluate metabolic activity, $6 \times 10^{3}$ cells were seeded into single wells of a 96-well plate. Cells were allowed to attach for $24 \mathrm{~h}$ and were subsequently exposed to either radiation, galunisertib, a combination of both or $0.16 \%$ DMSO which served as the control. For combination experiments, cells were exposed to different doses of irradiation, ranging from 2 to $8 \mathrm{~Gy}$. Subsequently, metabolic activity was evaluated using a colorimetric resazurin-based redox assay as described previously [12]. In brief, resazurin sodium salt stock at a concentration of $570 \mu \mathrm{M}$ was diluted at $1: 10 \mathrm{v} / \mathrm{v}$ in the cell culture medium and cells were exposed for $60 \mathrm{~min}$. Absorbance was measured at $570 \mathrm{~nm}$ using a microplate reader (Tecan Spark ®, Tecan Group Ltd., Maennedorf, Switzerland).

\section{Wound-healing assay}

$15 \times 10^{5}$ cells of each cell line were seeded in each well of a 24 well plate. After cells reached $95-100 \%$ confluency, they were exposed to either galunisertib, radiation or a combination of both. DMSO-treated cells served as a control group. A handmade scratch was produced with a $200 \mu \mathrm{l}$ pipette tip. The scratch was photographed at $0 \mathrm{~h}$ and $24 \mathrm{~h}$ using a microplate reader (Tecan Spark ®, Tecan Group Ltd., Maennedorf, Switzerland) and measured using ImageJ for further analysis.

\section{Colony formation assay}

Colony formation assays were performed as described previously [13]. In brief, $5 \times 10^{2}$ (CAL27), $6 \times 10^{2}(\mathrm{FaDu})$ or $15 \times 10^{2}(\mathrm{SCC}-25)$ cells were plated in 6-well plates and incubated for $24 \mathrm{~h}$. Cells were then treated with galunisertib and/or irradiated with $4 \mathrm{~Gy}$. After $72 \mathrm{~h}$, the drug-containing medium was replaced by a drug-free medium. After further 10 days, cells were washed with phosphate-buffered saline and photographed using a microplate reader (Tecan Spark ®, Tecan Group Ltd., Maennedorf, Switzerland). Colonies consisting of more than 50 cells were regarded as survivors and automatically counted using ImageJ. The surviving fractions were normalized to untreated controls.

\section{Flow cytometry}

HNSCC cell lines were seeded at $1 \times 10^{6}$ per well in 6-well plates. Cells were treated with either galunisertib, radiation, a combination of both or DMSO control $24 \mathrm{~h}$ after seeding. After $72 \mathrm{~h}$ of incubation, the cell cycle was analysed using DAPI staining and fluorescence-activated cell sorting (FACS) analysis (LSR Fortessa, BD Bioscience, USA).

\section{Statistical analysis}

All results represent three independent experiments and are reported as mean \pm SD. Statistical analysis was performed using GraphPad Prism for Mac Version 8 (GraphPad Software, LLC.). Statistical significance of differences between groups was determined using two-way-ANOVA and Tukey 
multiple comparison testing. A P-value below 0.05 was considered significant. Combinatory drug effects were defined using the Bliss independence method based on the assumption of independent drug mechanisms. In brief, the combined effect $\left(\mathrm{E}_{\mathrm{T}}\right)$ expressed as the surviving fraction is modelled as the product of the individual effects with drug "A" $\left(\mathrm{E}_{\mathrm{A}}\right)$ and "B" $\left(\mathrm{E}_{\mathrm{B}}\right)$, computed by: $\mathrm{E}_{\mathrm{T}}=\mathrm{E}_{\mathrm{A}} \mathrm{x} \mathrm{E}_{\mathrm{B}}$ [14]. If the drug combination is similar to the expected combined effect $\left(\mathrm{E}_{\mathrm{AB}}=\mathrm{E}_{\mathrm{T}}\right)$, then the combination would be additive, if it is less than expected $\left(\mathrm{E}_{\mathrm{AB}}<\mathrm{E}_{\mathrm{T}}\right)$ it would be synergistic and if it is greater $\left(\mathrm{E}_{\mathrm{AB}}>\mathrm{E}_{\mathrm{T}}\right)$ than it would be antagonistic [15].

\section{Results}

\section{Galunisertib shows heterogeneous effects on metabolic activity in HNSCC cell lines}

To determine the effect of galunisertib on metabolic activity, a resazurin-based reduction assay was performed after exposure of HNSCC cell lines to galunisertib for $72 \mathrm{~h}$. Vector $(0.16 \%$ DMSO) treated cells served as control. Galunisertib showed different effects on metabolic activity in all three tested HNSCC cell lines. While CAL27, a tongue carcinoma cell line, showed no effect (Fig. 1a), $\mathrm{FaDu}$, a hypopharyngeal SCC cell line, showed significantly reduced activity at $40 \mu \mathrm{M}$ (metabolic activity: $80 \%$ vs. $100 \%, \mathrm{P}<0.001$, Fig. $1 \mathrm{~b}$ ). The second tongue carcinoma cell line, namely SCC- 25 , showed an increased activity in response to galunisertib exposure which was highest at $20 \mu \mathrm{M}$, reaching $138 \%$, and declined to $115 \%$ at $40 \mu \mathrm{M}$ compared to control $(\mathrm{P}<0.05$, Fig. 1c). Radiation single treatment decreased metabolic activity in all three cell lines in a dose-dependent manner, with the strongest effect in CAL27, followed by FaDu and SCC-25 after a radiation dose of 8 Gy (Fig. 1 a-c, all $\mathrm{P}<0.001$ ). In combination treatment, galunisertib showed a significant additional reduction of metabolic activity at a concentration of $40 \mu \mathrm{M}$ in FaDu together with 2 and 4 Gy (60 vs. $80 \%$ and 56 vs. $67 \%$, respectively, $\mathrm{P}<0.001$ ), but no additional effect could be observed at $8 \mathrm{~Gy}(\mathrm{P}=0.974)$. No significant effect of combination treatment could be observed in CAL27. For SCC-25, the increased metabolic activity observed in galunisertib single treatment was also present in combination with radiation, increasing at 8 Gy from 62 to $87 \%$ in combination with $40 \mu \mathrm{M}$ galunisertib $(\mathrm{P}<0.001$, Fig. 1c $)$.

\section{Galunisertib reduces HNSCC cell migration}

To study the impact of galunisertib on cell migration, a wound-healing assay was performed as described previously [16]. Galunisertib single treatment inhibited migratory capabilities in all tested cell lines (Fig. 2). Radiation single treatment also reduced cell migration in all cell lines in a dose-dependent manner, with the strongest effect in CAL27, followed by SCC-25 and FaDu. In combination treatment, $40 \mu \mathrm{M}$ galunisertib showed an additive effect in CAL27 (gap closure at $8 \mathrm{~Gy}+40 \mu \mathrm{M}$ vs. $0 \mathrm{~Gy}+40 \mu \mathrm{M}$.: 14 vs. $44 \%, \mathrm{P}<0.001)$ and $\mathrm{SCC}-25(18$ vs. $33 \%, \mathrm{P}=0.015$, Fig. 2a,c) while the effect of combination treatment was antagonistic in $\mathrm{FaDu}$ (39 vs. 47\%, P=0.882, Fig. 2b).
CAL 27

a

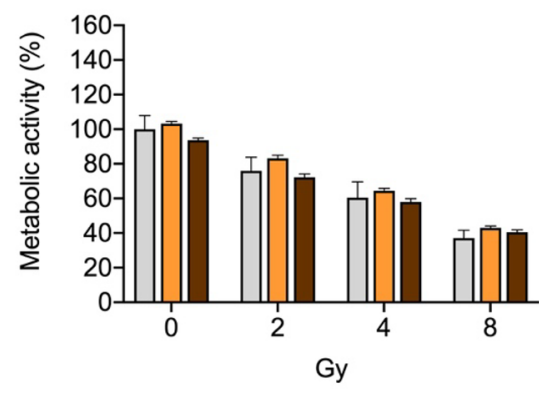

b

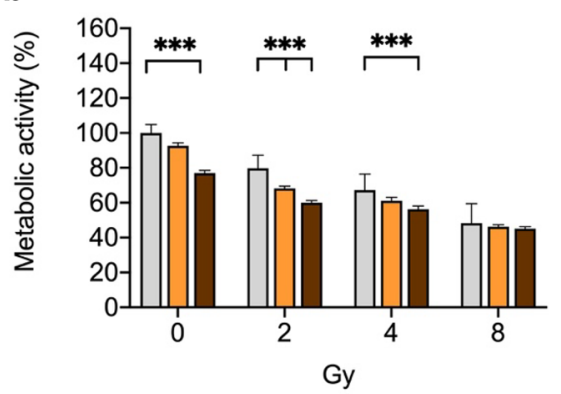

FaDu

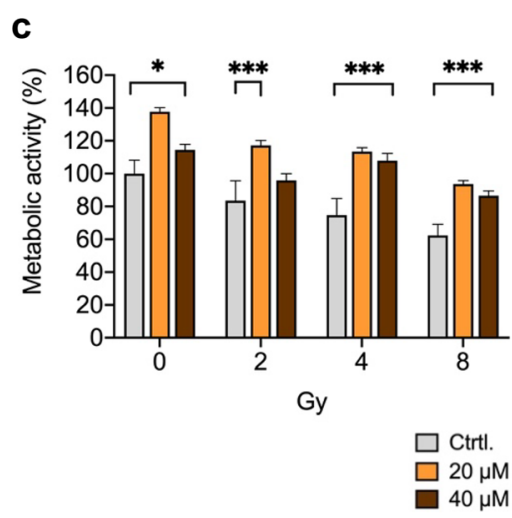

Fig. 1 Galunisertib decreased metabolic activity of $\mathrm{FaDu}$ cells, while it increased in SCC-25 and was unaffected in Cal27 cells after $72 \mathrm{~h}$ of exposure. Combination treatment of radiation with galunisertib did not affect metabolic activity in Cal27, but significantly decreased in $\mathrm{FaDu}$ at 2 and $4 \mathrm{~Gy}$. In SCC-25, galunisertib antagonized the effect of radiation on metabolic activity. Bar graphs are mean $+\mathrm{SD}, * \mathrm{p}<0.05$, $* * * \mathrm{p}<0.001$. Data represents metabolic activity as percentage of vector-treated control for three independent experiments including 6 replicates per condition 

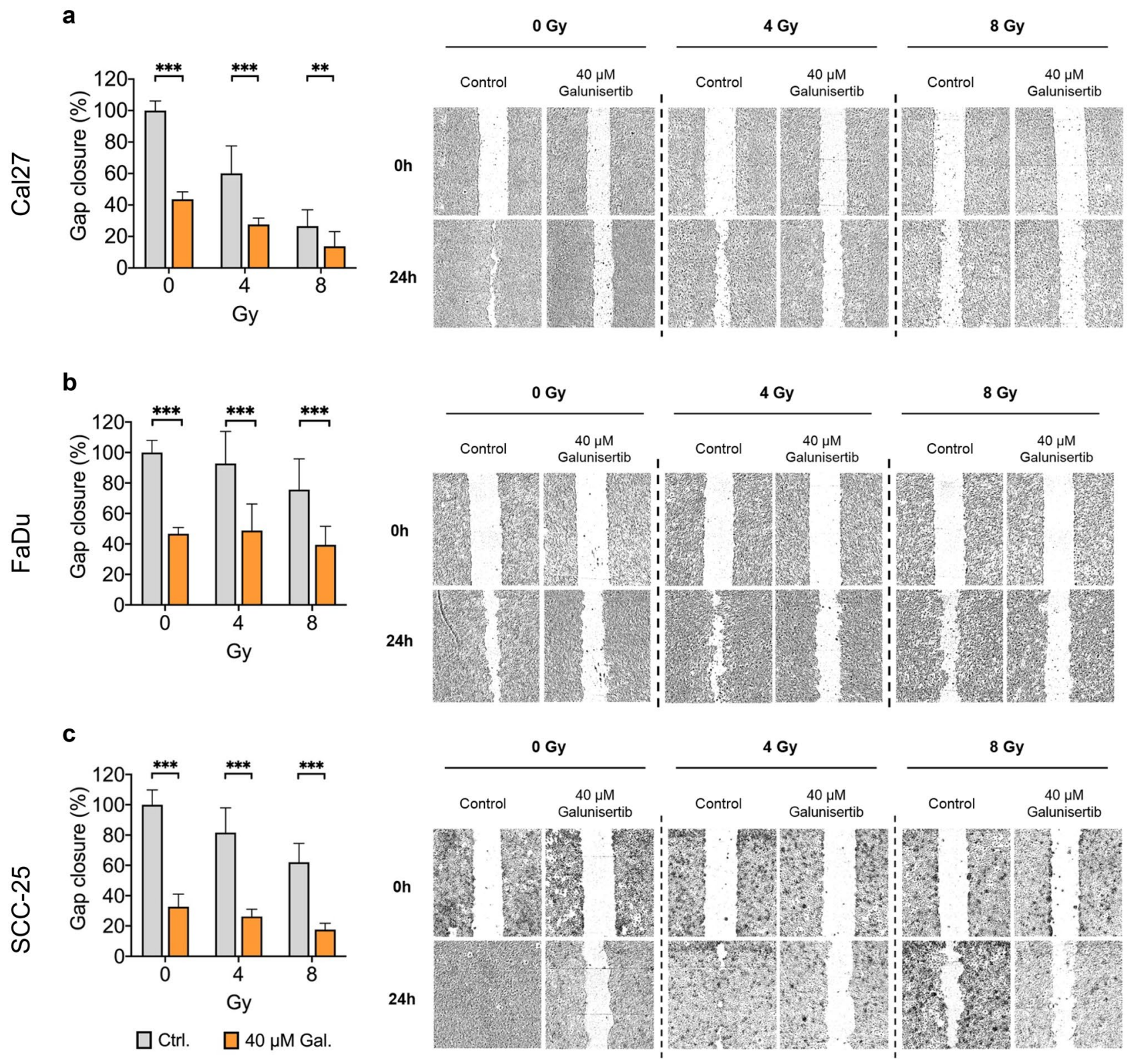

Fig. 2 Galunisertib treatment showed an additive effect on cell migration in combination with radiation for CAL27 and SCC-25 $(\mathrm{a}+\mathrm{c})$. Bar graphs are mean $+\mathrm{SD}, * * \mathrm{p}<0.01, * * * \mathrm{p}<0.001$. Data represents

\section{Galunisertib reduces clonogenic survival in SCC-25}

To test for the effect of galunisertib on clonogenic survival, colony formation assays were performed. Galunisertib single treatment showed a significant reduction in clonogenic survival of SCC-25 cells by $50 \%$ ( $\mathrm{P}<0.001)$, but did not affect clonogenic survival in CAL27 or FaDu. Radiation single treatment reduced clonogenic survival in all tested cell lines, with the strongest effect in SCC-25 (Fig. 3c). In combination with radiation, an additive effect could be observed for SCC25 (percent colonies formed at $40 \mu \mathrm{M}$ vs. $4 \mathrm{~Gy}+40 \mu \mathrm{M}, 47$ gap closure as a percentage of control for three independent experiments including 6 replicates per condition

vs. $10 \%, \mathrm{P}<0.001$, Fig. 3. c, d), while no radiosensitizing effect was observed in CAL27 or FaDu (Fig. 3a,b).

\section{Galunisertib affects cell cycle progression in SCC-25}

We used flow cytometry for DAPI-labelled DNA content to investigate the effects of galunisertib adjuvant to radiation on cell cycle progression. Cells were treated for $72 \mathrm{~h}$ with either $40 \mu \mathrm{M}$ galunisertib, radiation or a combination of both. DMSO treated cells served as control. Galunisertib single treatment showed a reduction of cells in $\mathrm{G}_{1}$ phase for SCC-25 (Ctrl. vs. 
Fig. 3 Galunisertib did not affect clonogenic survival in CAL27 or FaDu (a,b), but reduced clonogenic survival in SCC-25 and showed an additive effect in combination with radiation treatment (c). Representative images of the analysis for SCC-25 (d). Bar graphs are mean $+\mathrm{SD}$, ** $\mathrm{p}<0.01, * * * \mathrm{p}<0.001$. Data represent the percentage of colonies normalized to control for three independent experiments including three replicates per condition a

CAL27

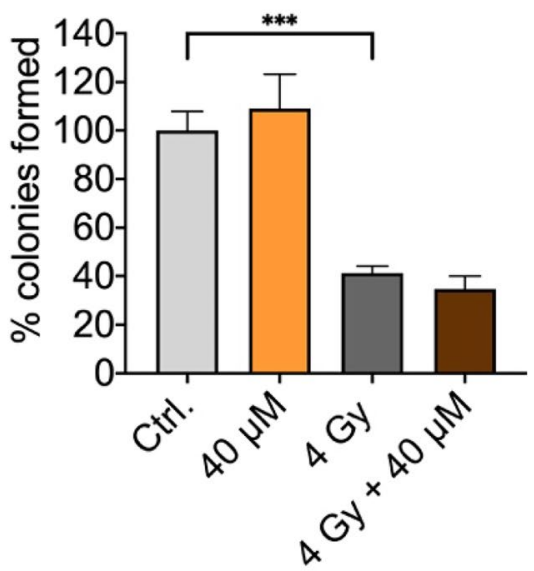

C

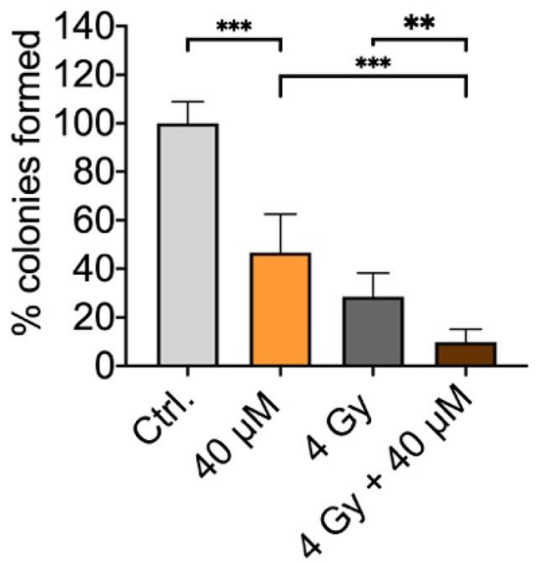

b FADU

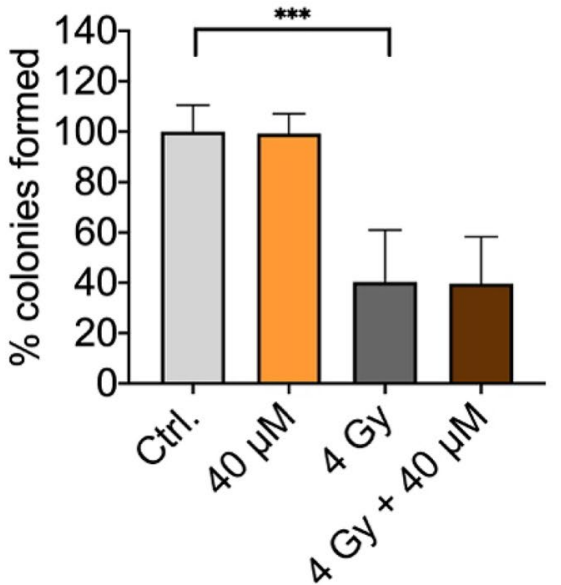

d

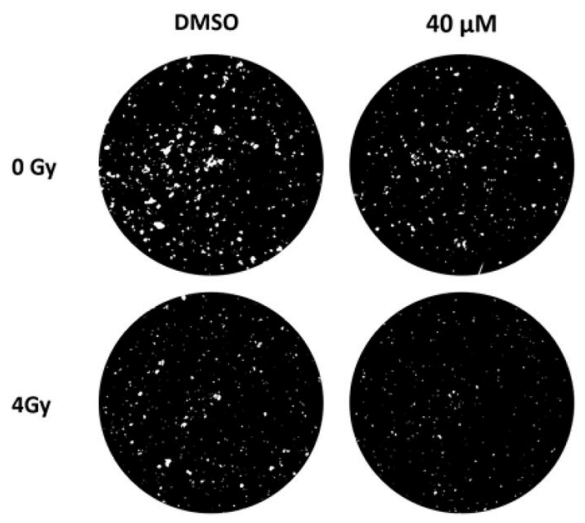

$40 \mu \mathrm{M}, 61 \%$ vs. $44 \%, \mathrm{P}=0.019)$ and a subsequent increase in $\mathrm{S}$ and $\mathrm{G}_{2}$ phase, although this difference was not statistically significant. Galunisertib single treatment did not affect cell cycle distribution in CAL27 or FaDu. While radiation treatment significantly decreased cells in $\mathrm{G}_{1}$ phase in CAL27 (Ctrl. vs. $4 \mathrm{~Gy}, 56 \%$ vs. $36 \%, \mathrm{P}<0.001)$ and FaDu (Ctrl. vs. $4 \mathrm{~Gy}$, $63 \%$ vs. $51 \%, \mathrm{P}<0.001)$ it did not significantly decrease cells in $\mathrm{G}_{1}$ phase in SCC-25. However, in combination treatment, an additive effect could be observed in SCC-25, further decreasing cells in $\mathrm{G}_{1}$ phase from 54 to $35 \%$ (8 Gy vs. $8 \mathrm{~Gy}+40 \mu \mathrm{M}$ respectively, Fig. 4c, $\mathrm{P}=0.006$ ).

\section{Discussion}

This is the first study to describe the in vitro effect of galunisertib in head and neck squamous cell carcinoma. Analysing three head and neck squamous cell carcinoma cell lines using standard preclinical assays, we observed varying antineoplastic effects in all tested cell lines at clinically relevant drug concentrations.

Radiation therapy is a cornerstone of treatment in a majority of patients suffering from head and neck 


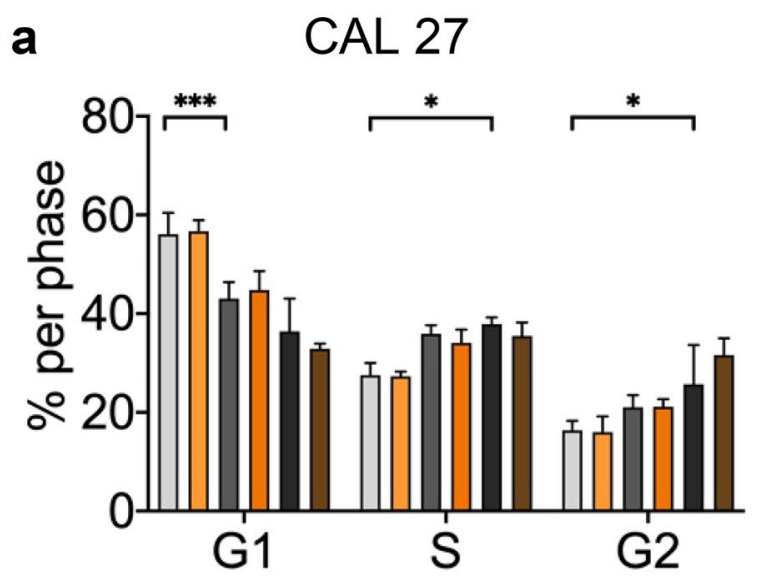

C

SCC-25

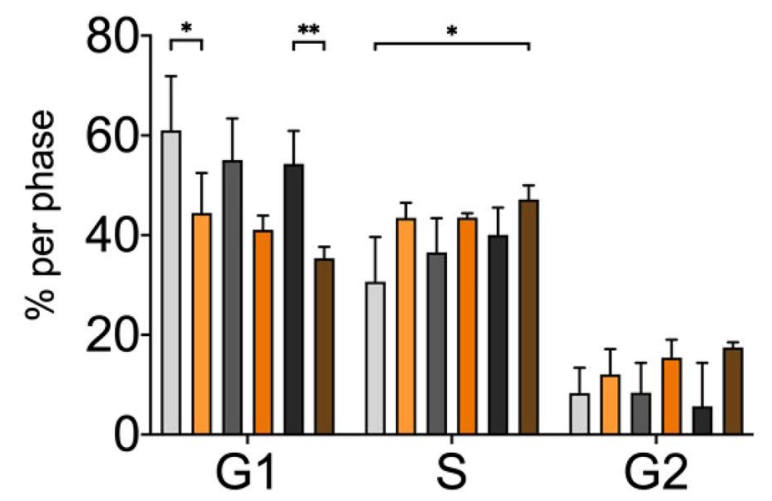

b

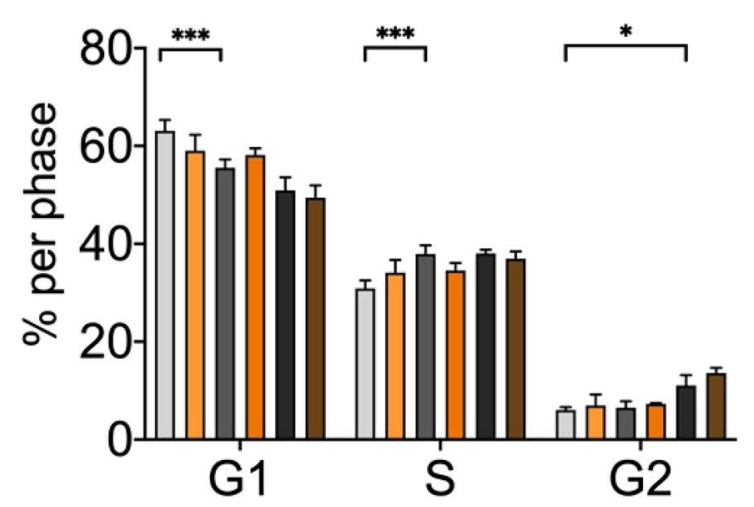

Fig. 4 All cell lines showed a shift from $G_{1}$ towards $S$ phase in response to radiation $(a, b, c)$. Galunisertib significantly reduced SCC25 in $G_{1}$ phase after single and combination treatment and increased cells in $\mathrm{S}$ phase in combination with radiation $(\mathrm{c}, \mathrm{d})$. Bar graphs are d
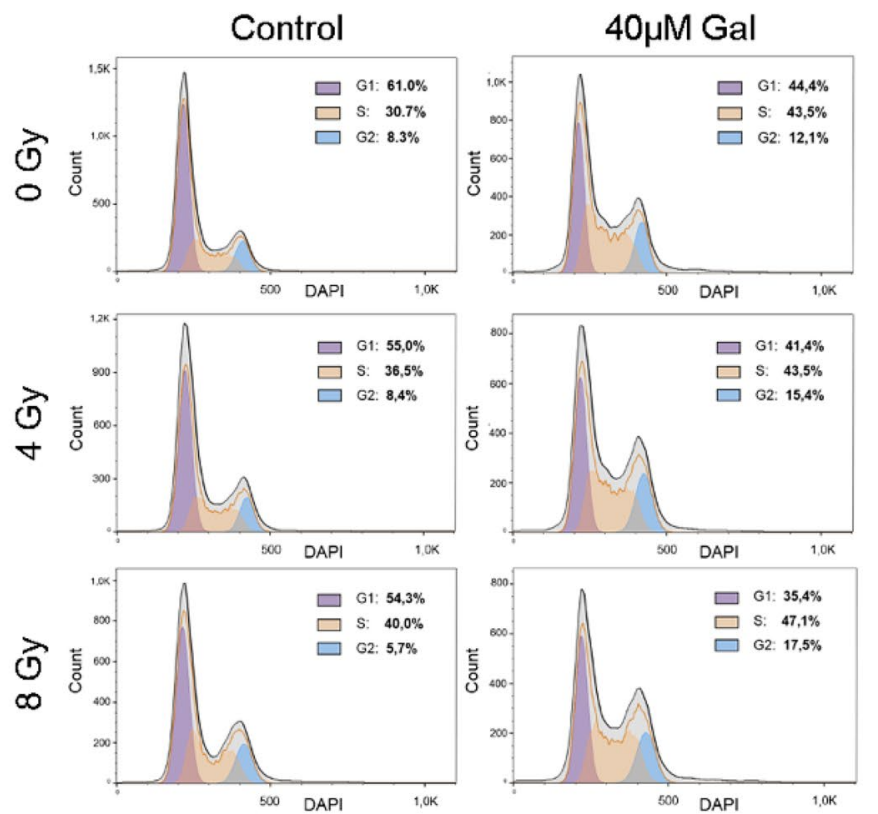

squamous cell carcinoma. Radioresistance, therefore, poses a major problem, which could be potentially abrogated using radiosensitizing agents. Research into the molecular mechanisms of radioresistance has led to the identification of many potential targets [17]. Transforming growth factor-beta (TGF- $\beta$ ) has emerged as such a target [18] and the availability of the small molecule TGF- $\beta$ receptor I inhibitor galunisertib, which is currently under clinical investigation as an antineoplastic agent, has led us to investigate its radiosensitizing potential in head and neck squamous cell carcinoma. mean $+\mathrm{SD}, * \mathrm{p}<0.05, * * \mathrm{p}<0.01, * * * \mathrm{p}<0.001$. Data represent the percentage of cells in each respective cell cycle phase for three independent experiments including three replicates per condition

Since its first demonstration of antitumor effects in xenograft models of non-small lung cancer and breast cancer [19], galunisertib has progressed through phase I and II clinical trials and is one of the most advanced candidates among small molecule TGF- $\beta$ inhibitors [20]. Clinical studies investigating galunisertib in hepatocellular carcinoma [21] and pancreatic cancer [22] have shown improved outcome of the treatment group. Most importantly, various clinical trials reported a safe toxicity profile with no dose-limiting events [20]. Clinical trials in phase I and I/II investigating the combination of galunisertib with radiation 
treatment in hepatocellular carcinoma (NCT02906397) and malignant glioma (NCT01220271), respectively, are underway. Interestingly, we could not find any in vitro studies that investigated the combination of galunisertib with radiation. However, a study by Hardee et al. has shown increased sensitivity to radiation therapy in glioblastoma treated with the TGF-B receptor I inhibitor LY364947 [23]. Furthermore, Yang et al. have shown that another TGF- $\beta$ receptor inhibitor (LY2109761) increased radiosensitivity in gastric cancer in vitro and in vivo [24], highlighting the expected potential of this treatment combination.

Here, we aimed at investigating the effect of galunisertib at clinically relevant doses. We, therefore, searched the literature for studies investigating the plasma concentration of galunisertib in clinical trials first and found that patients who received a dose of $150 \mathrm{mg}$ twice daily reached a plasma concentration of $\sim 10,000 \mathrm{ng} / \mathrm{ml}$ (corresponding to $\sim 27 \mu \mathrm{M}$ ) [25]. We thus subsequently prespecified to investigate galunisertib at concentrations of 20 and $40 \mu \mathrm{M}$, since higher serum concentrations might not be achievable in the clinical setting. Notably, at comparable concentrations of up to $10 \mu \mathrm{M}$, galunisertib showed potent and selective inhibition of SMAD 2/3 phosphorylation in hepatocellular carcinoma cells [26] and anaplastic carcinoma cells [27].

We observed the strongest effects of galunisertib in SCC-25. Cell migration and clonogenic survival were significantly inhibited by single treatment and showed additive effects in combination with radiation, suggesting a radiosensitizing antineoplastic effect. Surprisingly, galunisertib treatment had a stimulating effect on metabolic activity, a surrogate marker for cell proliferation, in SCC25 , which also antagonized the inhibitory effect of radiation treatment. We furthermore observed a cell cycle shift with a significant reduction of cells in $\mathrm{G}_{1}$ phase. This could potentially be explained by an inhibition of TGF- $\beta$ mediated $\mathrm{G}_{1}$ phase arrest, which is often lost in malignant cells [28], but appears to be still intact in SCC-25. Indeed, it has been shown that TGF- $\beta$ signaling intermediates can remain intact and activate TGF- $\beta$ responsive promoters in some HNSCC cell lines [29]. It would appear that the increase in metabolic activity at $72 \mathrm{~h}$ could be associated with this effect, emphasizing the dichotomous role of TGF- $B$ in tumor progression [30]. Interestingly, a recent publication by Oshimori et al. identified TGF-ß-responding SCCs that displayed hallmarks of malignancy during a slow-cycling proliferation. Interestingly, those cells showed better protection against DNA damaging agents [31]. An inhibition of this mechanism might therefore explain more efficient radiation-induced DNA damage. In the other two tested cell lines, drug response was not as distinct. In $\mathrm{FaDu}$, we could observe a reduction in metabolic activity and cell migration after galunisertib treatment. In CAL27, an inhibitory effect could be observed for cell migration after galunisertib treatment with an additive effect with radiation. Consistent with our results, galunisertib showed inhibitory effects on cell migration in primary cholangiocarcinoma cells [32] and hepatocellular carcinoma [26] at comparable concentrations of 50 and $10 \mu \mathrm{M}$, respectively. Furthermore, a study by Zhang et al. showed a reduction in cell viability in ovarian cancer cells in vitro, however, at concentrations above $100 \mu \mathrm{M}$ [33].

Mechanistically, the observed differences between cell lines could be explained by different degrees of TGF- $\beta$ escape. Martin et al. have shown that CAL27 harbour an inactivating SMAD4 mutation, which is strictly required for downstream signaling of TGF- $\beta$ receptor I [34]. FaDu has been shown to be SMAD4-deficient [35]. Notably, galunisertib has shown high selectivity for the TGF- $\beta$ pathway and potent inhibition of TGF- $\beta$ mediated SMAD2/3 phosphorylation [36]. This poses the question of the underlying mechanism of observed differences between CAL27 and FaDu. Interestingly, while CAL27 remained insensitive to TGF- 3 stimulation after SMAD4 re-expression in a study by Martin et al. [34], suggesting TGF- $\beta$ receptor alterations, $\mathrm{FaDu}$ showed partial restoration of TGF- $\beta$ responsiveness in a study by Hummer et al. [35]. In a subsequent analysis of tissue microarrays for SMAD4 expression, Martin et al. found detectable SMAD4 expression in $\sim 82 \%$ of HNSCC patients by immunohistochemistry, suggesting that a substantial patient population could potentially benefit from a TGF- $\beta$ receptor inhibitor therapy.

Here, we explored the effect of TGF- $\beta$ receptor I inhibition in HNSCC monoculture, neglecting the paracrine signaling observed in the tumor microenvironment. However, autocrine TGF- $\beta$ potentiation appears to play an important role in tumor progression and could explain the observed effects. [37]. Therefore, our in vitro results cannot be directly applied to predict the response of HNSCC to Galunisertib treatment in vivo. The evident importance of the tumor microenvironment in TGF- $\beta$ signaling calls for more advanced cancer models, including cells of the tumor stroma, in future studies [38]. Notably, similar studies investigating the effect of Galunisertib both in vitro and in vivo, found consistent antineoplastic effects [33, $39,40]$. It can therefore reasonably be assumed that the observed effect of Galunisertib on HNSCC cell lines might also be relevant in vivo.

In conclusion, we demonstrated that galunisertib shows significant radiosensitizing and antineoplastic effects in one out of three tested HNSCC cell lines in vitro. This observation correlates with evidence for TGF- $\beta$ signaling alterations in the two less responsive cell lines, therefore providing a rationale for further investigation of this new drug. 
Acknowledgements We thank Univ.-Doz. Dr. Aner Gurvitz (Center for Physiology and Pharmacology, Medical University of Vienna) for proofreading of the manuscript.

Authors' contributions BJJ, GH and KM conceived the concept, designed and planned the experiments. TL, MH and JS performed the experiments. TL and LK performed the data analysis. TL, MH and LK generated the graphs. BJJ, TL and NJC wrote the manuscript. All authors read and approved the manuscript before submission.

Funding Open access funding provided by Medical University of Vienna. This work was supported by the Medical Scientific Fund of the Mayor of the City of Vienna (grant no. 17023)

\section{Declarations}

Ethics approval and consent to participate Not applicable.

Informed consent Not applicable.

Consent for publication Not applicable.

Research involving Human Participants and/or Animals Not applicable.

Conflicts of interest All authors declare no conflict of interest.

Open Access This article is licensed under a Creative Commons Attribution 4.0 International License, which permits use, sharing, adaptation, distribution and reproduction in any medium or format, as long as you give appropriate credit to the original author(s) and the source, provide a link to the Creative Commons licence, and indicate if changes were made. The images or other third party material in this article are included in the article's Creative Commons licence, unless indicated otherwise in a credit line to the material. If material is not included in the article's Creative Commons licence and your intended use is not permitted by statutory regulation or exceeds the permitted use, you will need to obtain permission directly from the copyright holder. To view a copy of this licence, visit http://creativecommons.org/licenses/by/4.0/.

\section{References}

1. Rivera C, Venegas B (2014) Histological and molecular aspects of oral squamous cell carcinoma (Review). Oncol Lett 8(1):711. https://doi.org/10.3892/ol.2014.2103 [published Online First: Epub Date]

2. Bray F, Ferlay J, Soerjomataram I, Siegel RL, Torre LA, Jemal A (2018) Global cancer statistics 2018: GLOBOCAN estimates of incidence and mortality worldwide for 36 cancers in 185 countries. CA Cancer J Clin 68(6):394-424. https://doi.org/10.3322/ caac. 21492 [published Online First: Epub Date]

3. Disease GBD, Injury I, Prevalence C (2016) Global, regional, and national incidence, prevalence, and years lived with disability for 310 diseases and injuries, 1990-2015: a systematic analysis for the Global Burden of Disease Study 2015. Lancet 388(10053):1545-602. https://doi.org/10.1016/S0140-6736(16) 31678-6 [published Online First: Epub Date]

4. Chow LQM (2020) Head and Neck Cancer. N Engl J Med 382(1):60-72. https://doi.org/10.1056/NEJMra1715715 [published Online First: Epub Date]
5. Perri F, Pacelli R, Della Vittoria Scarpati G et al (2015) Radioresistance in head and neck squamous cell carcinoma: Biological bases and therapeutic implications. Head Neck 37(5):763-70. https://doi.org/10.1002/hed.23837 [published Online First: Epub Date]

6. Yamamoto VN, Thylur DS, Bauschard M, Schmale I, Sinha UK (2016) Overcoming radioresistance in head and neck squamous cell carcinoma. Oral Oncol 63:44-51. https://doi.org/10.1016/j. oraloncology.2016.11.002 [published Online First: Epub Date]

7. Centurione L, Aiello FB (2016) DNA Repair and Cytokines: TGF-beta, IL-6, and Thrombopoietin as Different Biomarkers of Radioresistance. Front Oncol 6:175. https://doi.org/10.3389/ fonc.2016.00175 [published Online First: Epub Date]

8. Colak S, Ten Dijke P (2017) Targeting TGF-beta Signaling in Cancer. Trends Cancer 3(1):56-71. https://doi.org/10.1016/j. trecan.2016.11.008 [published Online First: Epub Date]

9. White RA, Malkoski SP, Wang XJ (2010) TGFbeta signaling in head and neck squamous cell carcinoma. Oncogene 29(40):5437-46. https://doi.org/10.1038/onc.2010.306 [published Online First: Epub Date]

10. Herbertz S, Sawyer JS, Stauber AJ et al (2015) Clinical development of galunisertib (LY2157299 monohydrate), a small molecule inhibitor of transforming growth factor-beta signaling pathway. Drug Des Devel Ther 9:4479-99. https://doi.org/10. 2147/DDDT.S86621 [published Online First: Epub Date]

11. Cui X, Shang S, Lv X, Zhao J, Qi Y, Liu Z (2019) Perspectives of small molecule inhibitors of activin receptorlike kinase in antitumor treatment and stem cell differentiation (Review). Mol Med Rep 19(6):5053-62. https://doi.org/10.3892/mmr.2019. 10209 [published Online First: Epub Date]

12. O'Brien J, Wilson I, Orton T, Pognan F (2000) Investigation of the Alamar Blue (resazurin) fluorescent dye for the assessment of mammalian cell cytotoxicity. Eur J Biochem 267(17):5421-6. https://doi.org/10.1046/j.1432-1327.2000.01606.x [published Online First: Epub Date]

13. Franken NA, Rodermond HM, Stap J, Haveman J, van Bree C (2006) Clonogenic assay of cells in vitro. Nat Protoc 1(5):23159. https://doi.org/10.1038/nprot.2006.339 [published Online First: Epub Date]

14. Keith CT, Borisy AA, Stockwell BR (2005) Multicomponent therapeutics for networked systems. Nat Rev Drug Discov 4(1):71-8. https://doi.org/10.1038/nrd1609 [published Online First: Epub Date]

15. Ryall KA, Tan AC (2015) Systems biology approaches for advancing the discovery of effective drug combinations. J Cheminform 7:7. https://doi.org/10.1186/s13321-015-00559 [published Online First: Epub Date]

16. Kadletz L, Thurnher D, Wiebringhaus R et al (2017) Role of cancer stem-cell marker doublecortin-like kinase 1 in head and neck squamous cell carcinoma. Oral Oncol 67:109-18. https:// doi.org/10.1016/j.oraloncology.2017.02.007 [published Online First: Epub Date]

17. Barker HE, Paget JT, Khan AA, Harrington KJ (2015) The tumour microenvironment after radiotherapy: mechanisms of resistance and recurrence. Nat Rev Cancer 15(7):40925. https://doi.org/10.1038/nrc3958 [published Online First: Epub Date]

18. Andarawewa KL, Paupert J, Pal A, Barcellos-Hoff MH (2007) New rationales for using TGFbeta inhibitors in radiotherapy. Int J Radiat Biol 83(11-12):803-11. https://doi.org/10.1080/ 09553000701711063 [published Online First: Epub Date]

19. Bueno L, de Alwis DP, Pitou C et al (2008) Semi-mechanistic modelling of the tumour growth inhibitory effects of LY2157299, a new type I receptor TGF-beta kinase antagonist, in mice. Eur J Cancer 44(1):142-50. https://doi.org/10.1016/j.ejca.2007.10. 008 [published Online First: Epub Date] 
20. de Gramont A, Faivre S, Raymond E (2017) Novel TGF-beta inhibitors ready for prime time in onco-immunology. Oncoimmunology 6(1):e1257453. https://doi.org/10.1080/2162402X.2016. 1257453 [published Online First: Epub Date]

21. Faivre S, Santoro A, Kelley RK et al (2019) Novel transforming growth factor beta receptor I kinase inhibitor galunisertib (LY2157299) in advanced hepatocellular carcinoma. Liver Int 39(8):1468-77. https://doi.org/10.1111/liv.14113 [published Online First: Epub Date]

22. Melisi D, Garcia-Carbonero R, Macarulla T et al (2019) TGFbeta receptor inhibitor galunisertib is linked to inflammation- and remodeling-related proteins in patients with pancreatic cancer. Cancer Chemother Pharmacol 83(5):975-91. https://doi.org/10. 1007/s00280-019-03807-4 [published Online First: Epub Date]

23. Hardee ME, Marciscano AE, Medina-Ramirez CM et al (2012) Resistance of glioblastoma-initiating cells to radiation mediated by the tumor microenvironment can be abolished by inhibiting transforming growth factor-beta. Cancer Res 72(16):4119-29. https://doi.org/10.1158/0008-5472.CAN-12-0546 [published Online First: Epub Date]

24. Yang T, Huang T, Zhang D et al (2019) TGF-beta receptor inhibitor LY2109761 enhances the radiosensitivity of gastric cancer by inactivating the TGF-beta/SMAD4 signaling pathway. Aging (Albany NY) 11(20):8892-910. https://doi.org/10.18632/aging. 102329 [published Online First: Epub Date]

25. Kelley RK, Gane E, Assenat E et al (2019) A Phase 2 Study of Galunisertib (TGF-beta1 Receptor Type I Inhibitor) and Sorafenib in Patients With Advanced Hepatocellular Carcinoma. Clin Transl Gastroenterol 10(7):e00056. https://doi.org/10.14309/ ctg.0000000000000056 [published Online First: Epub Date]

26. Dituri F, Mazzocca A, Fernando J et al (2013) Differential Inhibition of the TGF-beta Signaling Pathway in HCC Cells Using the Small Molecule Inhibitor LY2157299 and the D10 Monoclonal Antibody against TGF-beta Receptor Type II. PLoS One 8(6):e67109. https://doi.org/10.1371/journal.pone.0067109 [published Online First: Epub Date]

27. Yingling JM, McMillen WT, Yan L et al (2018) Preclinical assessment of galunisertib (LY2157299 monohydrate), a first-inclass transforming growth factor-beta receptor type I inhibitor. Oncotarget 9(6):6659-77. https://doi.org/10.18632/oncotarget. 23795 [published Online First: Epub Date]

28. Donovan J, Slingerland J (2000) Transforming growth factor-beta and breast cancer: Cell cycle arrest by transforming growth factorbeta and its disruption in cancer. Breast Cancer Res 2(2):116-24. https://doi.org/10.1186/bcr43 [published Online First: Epub Date]

29. Yan W, Vellucci VF, Reiss M (2000) Smad protein expression and activation in transforming growth factor-beta refractory human squamous cell carcinoma cells. Oncol Res 12(3):157-67. https:// doi.org/10.3727/096504001108747639 [published Online First: Epub Date]

30. Huang JJ, Blobe GC (2016) Dichotomous roles of TGF-beta in human cancer. Biochem Soc Trans 44(5):1441-54. https://doi.org/ 10.1042/BST20160065 [published Online First: Epub Date]
31. Oshimori N, Oristian D, Fuchs E (2015) TGF-beta promotes heterogeneity and drug resistance in squamous cell carcinoma. Cell 160(5):963-76.https://doi.org/10.1016/j.cell.2015.01.043 [published Online First: Epub Date]

32. Lustri AM, Di Matteo S, Fraveto A et al (2017) TGF-beta signaling is an effective target to impair survival and induce apoptosis of human cholangiocarcinoma cells: A study on human primary cell cultures. PLoS One 12(9):e0183932. https://doi.org/10.1371/ journal.pone.0183932 [published Online First: Epub Date]

33. Zhang Q, Hou X, Evans BJ, VanBlaricom JL, Weroha SJ, Cliby WA (2018) LY2157299 Monohydrate, a TGF-betaR1 Inhibitor, Suppresses Tumor Growth and Ascites Development in Ovarian Cancer. Cancers (Basel) 10(8). https://doi.org/10.3390/cancers10080260 [published Online First: Epub Date]

34. Martin D, Abba MC, Molinolo AA et al (2014) The head and neck cancer cell oncogenome: a platform for the development of precision molecular therapies. Oncotarget 5(19):8906-23. https:// doi.org/10.18632/oncotarget.2417 [published Online First: Epub Date]

35. Hummer BT, Bartlett C, Henry E, Weissman BE (2003) Expression of Smad4 in the FaDu cell line partially restores TGF-beta growth inhibition but is not sufficient to regulate fibronectin expression or suppress tumorigenicity. J Cell Physiol 194(3):289_ 302. https://doi.org/10.1002/jcp.10202 [published Online First: Epub Date]

36. Sawyer JS, Anderson BD, Beight DW et al (2003) Synthesis and activity of new aryl- and heteroaryl-substituted pyrazole inhibitors of the transforming growth factor-beta type I receptor kinase domain. J Med Chem 46(19):3953-6. https://doi.org/10.1021/ jm0205705 [published Online First: Epub Date]

37. Derynck R, Akhurst RJ, Balmain A (2001) TGF-beta signaling in tumor suppression and cancer progression. Nat Genet 29(2):11729. https://doi.org/10.1038/ng1001-117 [published Online First: Epub Date]

38. Zhang H, Xie C, Yue J et al (2017) Cancer-associated fibroblasts mediated chemoresistance by a FOXO1/TGFbeta1 signaling loop in esophageal squamous cell carcinoma. Mol Carcinog 56(3):1150-63. https://doi.org/10.1002/mc.22581 [published Online First: Epub Date]

39. Liu CC, Wu CL, Lin MX, Sze CI, Gean PW (2021) Disulfiram Sensitizes a Therapeutic-Resistant Glioblastoma to the TGF-beta Receptor Inhibitor. Int J Mol Sci 22(19). https://doi.org/10.3390/ ijms221910496 [published Online First: Epub Date]

40. Gao H, Bai P, Xiao L et al (2020) Mediator complex subunit 16 is down-regulated in papillary thyroid cancer, leading to increased transforming growth factor-beta signaling and radioiodine resistance. J Biol Chem 295(31):10726-40. https://doi.org/10.1074/jbc. RA119.012404 [published Online First: Epub Date]

Publisher's Note Springer Nature remains neutral with regard to jurisdictional claims in published maps and institutional affiliations. 